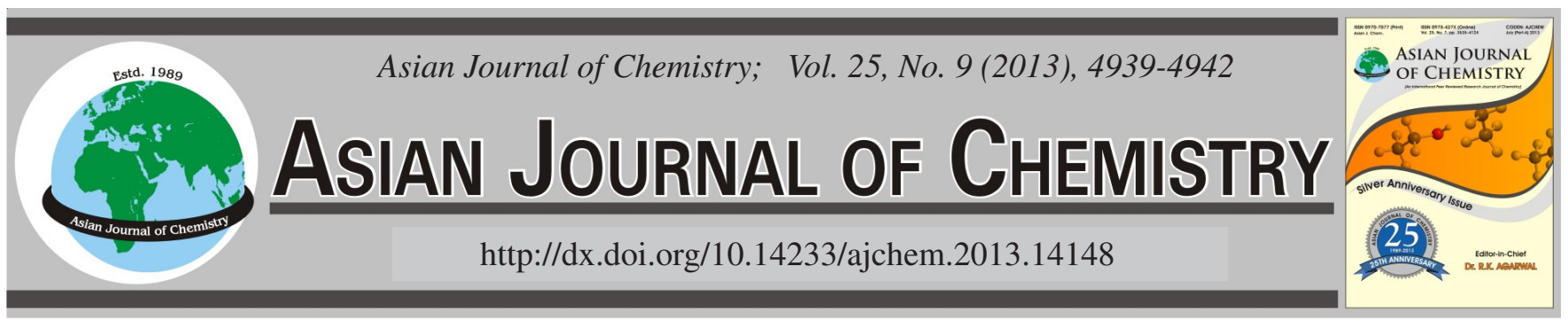

\title{
One-pot Synthesis of 2-Alkylthio-1,3,4-oxadiazole and Bis-(1,3,4-oxadiazole-2-yl)thio alkyl Derivatives from Acid Hydrazides and $\mathrm{CS}_{2}$
}

\section{Mohammad Soleiman-Beigi ${ }^{1, *}$, Mohammad Alikarami $^{2}$ and Tahereh HosseinZadeh ${ }^{3}$}

\author{
${ }^{1}$ Department of Chemistry, Faculty of Basic Science, Ilam University, P.O. Box 69315-516, Ilam, Iran \\ ${ }^{2}$ Department of Chemistry, Ilam Branch, Islamic Azad University, Ilam, Iran \\ ${ }^{3}$ Department of Chemistry, Faculty of Basic Science, Ilam University, Ilam, Iran \\ *Corresponding author: Fax: +98 841 2227022; E-mail: SoleimanBeigi@yahoo.com
}

(Received: 5 June 2012;

Accepted: 11 March 2013)

AJC-13108

An efficient and one-pot method for synthesis of 2-alkylthio-1,3,4-oxadiazole derivatives in mild conditions is described. Some novel derivatives such as bis-1,3,4-oxadiazole analogs are also synthesized.

Key Words: One-pot method, 2-Alkylthio-1,3,4-oxadiazole derivatives, Bis-1,3,4-oxadiazole analogs, Acid hydrazides.

ᄂ - - - - - - - - - - - - - - - - - - - - - - - - -

\section{INTRODUCTION}

Nowadays, one-pot multicomponent reactions have emerged as valuable tools in organic synthesis for reducing operative steps and enhancing synthesis efficiency ${ }^{1-3} \cdot 2$-Alkythio derivatives of 1,3,4-oxadiazole-2-thiols (thiones) are an important class of five-member heterocyclic compounds which have attracted much attention due to their applications as key intermediates in organic and inorganic synthesis ${ }^{4,5}$ and studies of biological activity ${ }^{6-11}$.

Theoretical studies and investigations shown that 1,3,4oxadiazole-2-thione derivatives are in equlibrium to their thiols tautomeric forms (Scheme-I) ${ }^{12-14}$.<smiles>[R]c1nnc(S)o1</smiles>

Scheme-I: Thiol-thione tautomerization of 1,3,4-oxadiazole-2-thione

Due to thiol-thione tautomeric equilibrium, their derivatives represent an important type of heterocyclic compound as key intermediates in the field of heterocyclic chemistry because of their potential multifunctional donor sites, via either exocyclic sulfur or endo cyclic nitrogen. Many methods are reported for $\mathrm{N}$-alkylation or $\mathrm{S}$-alkylation of $1,3,4$ oxadiazole-2-thiones in different mediums which, 1,3,4oxadiazole-2-thiones are generally used as the substrate after purification ${ }^{15-25}$. By far, the most common strategy for the synthesis of 1,3,4-oxadiazole-2-thiols involves the interaction of acid hydrazides with carbon disulfide in the presence of alcoholic potassium hydroxide ${ }^{26-30}$. Furthermore, in order to obtain pure 1,3,4-oxadiazole-2-thiols in the presence of a catalyst such as $\mathrm{KOH}$, the reaction mixture should be neutralized because the parent compound of 1,3,4-oxadiazole-2-thiol has acidic character $\left(\mathrm{pK}_{\mathrm{a}}=3.85\right)^{31}$. This makes the procedure rather difficult and reduces the yield of the product.

In the past few years, catalyst-free and one-pot reactions have been noted as important routes for the development of organic synthesis methodologies due to their environmentally friendly properties and their economic advantages ${ }^{32,33}$.

\section{EXPERIMENTAL}

All chemicals were purchased from Merck, Fluka and Aldrich chemical companies. Yields refer to isolated products. Melting points were determined by an Electrothermal 9100 apparatus and are uncorrected. The IR spectra were obtained on a FT-IR Hartman-Bomen spectrophotometer as $\mathrm{KBr}$ disks. The ${ }^{1} \mathrm{H}$ NMR (400 and $300 \mathrm{MHz}$ ) and ${ }^{13} \mathrm{C}$ NMR (100 and 75 $\mathrm{MHz}$ ) spectra were recorded on a Bruker Avance NMR spectrometer in $\mathrm{CDCl}_{3}$ solutions. Mass spectra were recorded on an Agilent Technology (HP) 5973 instrument (ionizing voltage $70 \mathrm{eV}$ ). Elemental analyses were done on a Carlo-Erba EA1110CHNO-S analyzer. The progress of the reaction was followed by TLC using silica-g el SIL G/ UV 254 plates. Products were characterized by comparing their physical and spectral data with those of the authentic samples.

General procedure for synthesis of 2-alkylthio-5-aryl1,3,4-oxadiazole derivatives: A mixture of acid hydraizde 
$(1.0 \mathrm{mmol})$ and carbon disulfide $(3.0 \mathrm{mmol})$ in $\mathrm{DMF}(2.0 \mathrm{~mL})$ was stirred for $15 \mathrm{~min}$ at room temperature, the mixture of reaction then was heated at $70{ }^{\circ} \mathrm{C}$ for appropriated time until complete ring closure ( $4 \mathrm{~h})$. After cooling to room temperature, alkyl halide $(1.2 \mathrm{mmol})$ and $\mathrm{Et}_{3} \mathrm{~N}(4 \mathrm{mmol})$ were added to mixture of reaction and stirred again at room temperature until completion of reaction as monitored by TLC (ethyl acetate: $n$-hexane (1:2). Then the solvent was evaporated in vacuo and the crude product was purified by preparative TLC (silica gel, eluent EtOAc: $n$-hexane 1:2) to obtain pure product.

Ethyl-[5-(4-chlorophenyl)-1,3,4-oxadiazol)-2-ylthio] acetate (Table-2, entry 2): m.p. $85-86{ }^{\circ} \mathrm{C}^{34}$. IR ( $\mathrm{KBr}, \mathrm{v}_{\max }$, $\left.\mathrm{cm}^{-1}\right)$ : 1736, 1602, 1200, 1088. ${ }^{1} \mathrm{H}$ NMR $\left(400 \mathrm{MHz}, \mathrm{CDCl}_{3}\right)$ : $\delta 1.31\left(\mathrm{t}, J=7.2 \mathrm{~Hz}, 3 \mathrm{H},-\mathrm{CH}_{3}\right), 4.12\left(\mathrm{~s}, 2 \mathrm{H},-\mathrm{SCH}_{2}\right), 4.27$ (q, $\left.J=7.2 \mathrm{~Hz}, 2 \mathrm{H},-\mathrm{OCH}_{2}\right), 7.49$ (d, $\left.J=8.4 \mathrm{~Hz}, 2 \mathrm{H}, \mathrm{Ar}-\mathrm{H}\right), 7.94$ $(\mathrm{d}, J=8.4 \mathrm{~Hz}, 2 \mathrm{H}, \mathrm{Ar}-\mathrm{H}) .{ }^{13} \mathrm{C} \mathrm{NMR}\left(100 \mathrm{MHz}, \mathrm{CDCl}_{3}\right): \delta$ $14.09,34.40,62.45,121.92,127.96,129.47,138.03,163.23$, $165.25,167.30$.

2-(Methylthio)-5-(4-methylphenyl)-1,3,4-oxadiazole (Table-2, entry 6): m.p. $50-51{ }^{\circ} \mathrm{C}$. IR ( $\left.\mathrm{KBr}, \mathrm{v}_{\max }, \mathrm{cm}^{-1}\right)$ : 1611 , 1477, 1087. ${ }^{1} \mathrm{H}$ NMR $\left(300 \mathrm{MHz}, \mathrm{CDCl}_{3}\right): \delta 2.42(\mathrm{~s}, 3 \mathrm{H}$, $\left.-\mathrm{CH}_{3}\right), 2.78$ (s, 3H, Ar- $\mathrm{CH}_{3}$ ), 7.30 (d, $\left.J=8.1,2 \mathrm{H}, \mathrm{Ar}-\mathrm{H}\right), 7.90$ $(\mathrm{d}, J=8.1,2 \mathrm{H}, \mathrm{Ar}-\mathrm{H}) .{ }^{13} \mathrm{C} \mathrm{NMR}\left(75 \mathrm{MHz}, \mathrm{CDCl}_{3}\right): \delta 14.66$, 21.63, 120.87, 126.61, 129.72, 142.17, 164.3, 165.60.

2-(Ethylylthio)-5-phenyl-1,3,4-oxadiazole (Table-2, entry 8): m.p. $46-48{ }^{\circ} \mathrm{C}$. IR $\left(\mathrm{KBr}, v_{\max }, \mathrm{cm}^{-1}\right): 1605,1086 .{ }^{1} \mathrm{H}$ NMR (400 MHz, $\left.\mathrm{CDCl}_{3}\right): \delta 1.53\left(\mathrm{t}, J=7.6 \mathrm{~Hz}, 3 \mathrm{H},-\mathrm{CH}_{3}\right)$, $3.33\left(\mathrm{q}, 2 \mathrm{H}, J=7.6 \mathrm{~Hz},-\mathrm{SCH}_{2}\right), 7.52(\mathrm{~m}, 3 \mathrm{H}, \mathrm{Ar}-\mathrm{H}), 8.02(\mathrm{~m}$, $2 \mathrm{H}, \mathrm{Ar}-\mathrm{H}) .{ }^{13} \mathrm{C} \mathrm{NMR}\left(100 \mathrm{MHz}, \mathrm{CDCl}_{3}\right): \delta$ 14.76, 27.04, 123.68, 126.61, 129,01, 131.57, 164.33, 165.66. MS (EI, 70 $\mathrm{eV}): \mathrm{m} / \mathrm{z}(\%)=206$ (95), 178 (42), 145 (67), 105 (100), 77 (84). Anal. calcd. for $\mathrm{C}_{10} \mathrm{H}_{10} \mathrm{~N}_{2} \mathrm{OS}$ : C, 58. 23; H, 4.89; N, 13.58. Found: C, 58. 10; H, 4.97; N, 13.70.

2-(Allylthio)-5-(4-chlorophenyl)-1,3,4-oxadiazole (Table-2, entry 9): m.p. $59-61^{\circ} \mathrm{C}$. IR $\left(\mathrm{KBr}, \nu_{\max }, \mathrm{cm}^{-1}\right)$ : 1640 , 1603, 1087. ${ }^{1} \mathrm{H}$ NMR $\left(300 \mathrm{MHz}, \mathrm{CDCl}_{3}\right): \delta 3.93(\mathrm{~d}, J=8.0$ $\left.\mathrm{Hz}, 2 \mathrm{H},-\mathrm{SCH}_{2}\right), 5.23(\mathrm{~d}, J=9.9,1 \mathrm{H}, \mathrm{C}=\mathrm{CH}), 5.40(\mathrm{~d}, J=$ 15.9, 1H, C=CH), $6.01(\mathrm{~m}, 1 \mathrm{H}, \mathrm{CH}=\mathrm{C}), 7.47(\mathrm{~d}, J=6.7,2 \mathrm{H}$, Ar-H), 7.94 (d, $J=6.7,2 \mathrm{H}, \mathrm{Ar}-\mathrm{H}) .{ }^{14} \mathrm{C} \mathrm{NMR}\left(75 \mathrm{MHz}, \mathrm{CDCl}_{3}\right)$ : $\delta 35.25,119.89,122.10,127.90,129.43,131.61,137.89$, 163.99, 165.02. MS (EI, $70 \mathrm{eV}): \mathrm{m} / \mathrm{z}$ (\%) 252 (28), 179 (18), 139 (100), 115 (51), 82 (54). Anal. calcd. for $\mathrm{C}_{11} \mathrm{H}_{9} \mathrm{~N}_{2} \mathrm{OSCl}$ : C, 52. 28; H, 3.59; N, 11.08. Found: C, 52. 11; H, 3.37; N, 11.22 .

2-(Benzylthio)-5-phenyl-1,3,4-oxadiazole (Table-2, entry 11): m.p. $92-94{ }^{\circ} \mathrm{C}$. IR $\left(\mathrm{KBr}, v_{\max }, \mathrm{cm}^{-1}\right): 1613,1585$ 1087. ${ }^{1} \mathrm{H}$ NMR (400 MHz, $\left.\mathrm{CDCl}_{3}\right): \delta 4.54\left(\mathrm{~s}, 2 \mathrm{H},-\mathrm{SCH}_{2}\right.$ ), 7.27-7.99 (m, 10H, Ar-H). ${ }^{13} \mathrm{C}$ NMR (100 MHz, $\left.\mathrm{CDCl}_{3}\right): \delta$ 36.85, 123.60, 126.67, 128.14, 128.84, 129.06, 129.20, 131.68, 135.64, 163.89, 165.84. MS (EI, $70 \mathrm{eV}): \mathrm{m} / \mathrm{z}$ (\%) 268 (12), 206 (76), 178 (30), 145 (63), 105 (100), 91 (45), 77 (85). Anal. calcd. for $\mathrm{C}_{15} \mathrm{H}_{12} \mathrm{~N}_{2} \mathrm{OS}$ : C, 67. 14; H, 4.51; N, 10.44. Found: C, 67. 31; H, 4.77; N, 10.21.

2-((5-(4-Chlorophenyl)-1,3,4-oxadiazole-2-yl)thio)acetic acid (Table-2, entry 12): m.p. $148-150^{\circ} \mathrm{C}$. IR ( $\left.\mathrm{KBr}, v_{\max }, \mathrm{cm}^{-1}\right)$ : 3200-2400, 1736, 1610. ${ }^{1} \mathrm{H}$ NMR (300 MHz, $\left.\mathrm{CDCl}_{3}\right) \delta 3.05$ (s, 2H, $\left.-\mathrm{SCH}_{2}\right), 7.47$ (d, $\left.J=8.43 \mathrm{~Hz}, 2 \mathrm{H}, \mathrm{Ar}-\mathrm{H}\right), 7.86$ (d, $J=$ $8.45 \mathrm{~Hz}, 2 \mathrm{H}, \mathrm{Ar}-\mathrm{H}), 13.02$ (s, 1H, COOH). ${ }^{13} \mathrm{C}$ NMR (100
$\left.\mathrm{MHz}, \mathrm{CDCl}_{3}\right): \delta 37.30,121.04,127.71,129.58,138.64$, 160.65, 163.96, 178.0. Anal. calcd. for $\mathrm{C}_{10} \mathrm{H}_{8} \mathrm{~N}_{2} \mathrm{O}_{3} \mathrm{~S}$ : C, 50 . 84; H, 3.41; N, 11.86. Found: C, 51. 08; H, 3.67; N, 12.04.

1,2-Bis((5-phenyl-1,3,4-oxadiazole-2-yl)thio) ethane (Table-2, entry 13): m.p. $122-125^{\circ} \mathrm{C}$. IR $\left(\mathrm{KBr}, \mathrm{v}_{\max }, \mathrm{cm}^{-1}\right)$ : 1604, 1087. ${ }^{1} \mathrm{H}$ NMR $\left(400 \mathrm{MHz}, \mathrm{CDCl}_{3}\right): \delta 3.86(\mathrm{~s}, 4 \mathrm{H}$, $\left.-\mathrm{CH}_{2}\right), 7.55(\mathrm{~m}, 6 \mathrm{H}, \mathrm{Ar}-\mathrm{H}), 8.04(\mathrm{~m}, 4 \mathrm{H}, \mathrm{Ar}-\mathrm{H}) .{ }^{13} \mathrm{C}$ NMR $\left(100 \mathrm{MHz}, \mathrm{CDCl}_{3}\right): \delta 36.54,124.60,126.51,128,01,131.55$, 164.03, 165.60. MS (EI, $70 \mathrm{eV}): \mathrm{m} / \mathrm{z}$ (\%) 382 (62), 205 (100), 145 (42), 105 (41), 77 (57). Anal. calcd. for $\mathrm{C}_{19} \mathrm{H}_{17} \mathrm{~N}_{4} \mathrm{O}_{2} \mathrm{~S}_{2}$ : C, 57. 41; H, 4.31; N, 14.10. Found: C, 57. 21; H, 4.57; N, 13.88.

\section{RESULTS AND DISCUSSION}

These issues have prompted us to investigate the synthesis of 2-alkylthio-1,3,4-oxadiazole in one-pot and mild solution conditions from acid hydrazides and carbon disulfide. In this work, we have explored an one-pot method for chemoselective synthesis of 2-alkylthio-1,3,4-oxadiazole derivatives from acid hydrazides (Scheme-II).

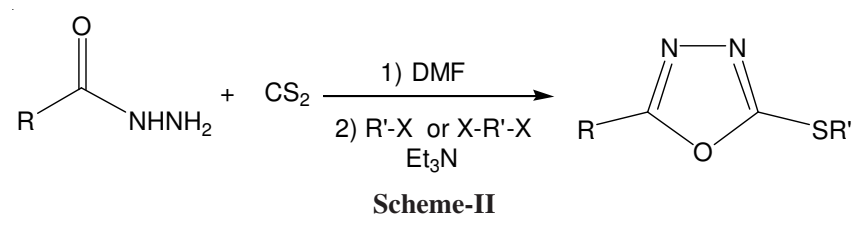

To optimize reaction conditions, reaction of benzhydrazide and $\mathrm{CS}_{2}$ at catalyst-free condition in DMF was studied as model reaction. The reaction mixture was stirred for $0.5 \mathrm{~h}$ in room temperature, then the temperature was increased to $70{ }^{\circ} \mathrm{C}$ for $3.5 \mathrm{~h}$. After completion of reaction, ethyl iodide we added to mixture of reaction at room temperature in the presence of some different bases as the results are presented in Table- 1 .

\begin{tabular}{|c|c|c|}
\hline \multicolumn{3}{|c|}{$\begin{array}{l}\text { TABLE-1 } \\
\text { SEEKING THE SUITABLE BASE FOR THE ONE-POT } \\
\text { SYNTHESIS OF 2-ETHYLTHIO-5-PHENYL-1,3,4- } \\
\text { OXADIAZOLE FROM BENZHYDRAZIDE IN 6 h }\end{array}$} \\
\hline Entry & Base & Yield $(\%)^{\mathrm{a}}$ \\
\hline 1 & None & - \\
\hline 2 & $\mathrm{KF} / \mathrm{Al}_{2} \mathrm{O}_{3}$ & 43 \\
\hline 3 & $\mathrm{TBAH}^{\mathrm{b}}{ }^{\mathrm{j}}$ & 68 \\
\hline 4 & $\mathrm{KOH}$ & 21 \\
\hline 5 & $\mathrm{NaOH}$ & 11 \\
\hline 6 & $\mathrm{Et}_{3} \mathrm{~N}$ & 98 \\
\hline 7 & DABCO & 80 \\
\hline
\end{tabular}

As shown in Table-1, this procedure dose not proceeds properly at the presence of strong bases such as $\mathrm{KF} / \mathrm{Al}_{2} \mathrm{O}_{3}$, $\mathrm{KOH}, \mathrm{NaOH}$ and TBAH (Table-1, entries 2-5). Furthermore, no product was observed in the base-free condition (Table-1, entry 1). While at the presence of organo bases such as $\mathrm{Et}_{3} \mathrm{~N}$ and DABCO, 2-ethylthio-5-phenyl-1,3,4-oxadiazole was obtained in high yields (Table-1, entries 6 and 7). Herein, $\mathrm{Et}_{3} \mathrm{~N}$ was selected as suitable and accessible base.

To test the generality of this procedure in optimized conditions, we have examined the reaction of acid hydrazides with $\mathrm{CS}_{2}$ and a variety kind of alkyl halides such as alkyl, 


\begin{tabular}{|c|c|c|c|c|c|}
\hline Entry & R- & $\mathrm{R}^{\prime} \mathrm{X}$ & Product & Time (min) & Yield $(\%)^{\mathrm{a}}$ \\
\hline 1 & $\mathrm{C}_{6} \mathrm{H}_{5}$ & $\mathrm{BrCH}_{2} \mathrm{CO}_{2} \mathrm{Et}$ & & 120 & 96 \\
\hline 2 & 4- $\mathrm{Cl}-\mathrm{C}_{6} \mathrm{H}_{4}$ & $\mathrm{BrCH}_{2} \mathrm{CO}_{2} \mathrm{Et}$ & & 50 & 95 \\
\hline 3 & 2-Cl- $\mathrm{C}_{6} \mathrm{H}_{4}$ & $\mathrm{BrCH}_{2} \mathrm{CO}_{2} \mathrm{Et}$ & & 50 & 90 \\
\hline 4 & $3-\mathrm{NC}_{5} \mathrm{H}_{4}$ & $\mathrm{BrCH}_{2} \mathrm{CO}_{2} \mathrm{Et}$ & & 60 & 75 \\
\hline 5 & $\mathrm{C}_{6} \mathrm{H}_{5}$ & $\mathrm{CH}_{3} \mathrm{I}$ & & 90 & 87 \\
\hline 6 & 4- $\mathrm{CH}_{3}-\mathrm{C}_{6} \mathrm{H}_{4}$ & $\mathrm{CH}_{3} \mathrm{I}$ & & 50 & 71 \\
\hline 7 & 4-Cl- $\mathrm{C}_{6} \mathrm{H}_{4}$ & $\mathrm{CH}_{3} \mathrm{I}$ & & 40 & 86 \\
\hline 8 & $\mathrm{C}_{6} \mathrm{H}_{5}$ & $\mathrm{CH}_{3} \mathrm{CH}_{2} \mathrm{I}$ & & 45 & 98 \\
\hline 9 & 4-Cl- $\mathrm{C}_{6} \mathrm{H}_{4}$ & $\mathrm{CH}_{2}=\mathrm{CHCH}_{2} \mathrm{Br}$ & & 40 & 97 \\
\hline 10 & 2-OH- $\mathrm{C}_{6} \mathrm{H}_{4}$ & $\mathrm{CH}_{2}=\mathrm{CHCH}_{2} \mathrm{Br}$ & & 50 & 75 \\
\hline 11 & $\mathrm{C}_{6} \mathrm{H}_{5}$ & $\mathrm{PhCH}_{2} \mathrm{Cl}$ & & 40 & 75 \\
\hline 12 & 4-Cl- $\mathrm{C}_{6} \mathrm{H}_{4}$ & $\mathrm{ClCH}_{2} \mathrm{CO}_{2} \mathrm{H}$ & & 55 & 72 \\
\hline 13 & $\mathrm{C}_{6} \mathrm{H}_{5}$ & $\mathrm{BrCH}_{2} \mathrm{CH}_{2} \mathrm{Br}$ & & 70 & 93 \\
\hline
\end{tabular}


$\alpha$-halo acid and ester, benzyl and allyl, as results are shown in Table-2.

When benzhydrazide was treated with alkyl halides under optimized reaction conditions, corresponding products were obtained in good to excellent yields (Table-2, entries 1, 5,8 and 11). Similarly, reactions of aryl substituted acid hydrazides (para-methyl, para-chloro, ortho-chloro and orthohydroxy aryl acid hydrazides) with methyl iodide (Table-2, entries 6 and 7), $\alpha$-bromo ester (Table-2, entries 2 and 3), $\alpha$ - chloro acid (Table-2, Entry 12) and allyl bromide (Table-2, entries 9 and 10) gave the products in high yields (71-97\%). In addition, from treating of 3-nicotinic hydrazide acid as a hetroaryl acid hydrazide with $\alpha$-bromo ester (Table-2, entry 4 ) corresponded product was yielded very well.

The procedure, was also examined for synthesis of bis1,3,4-oxadiazole compounds via reaction of benzhydrazde (2 mmol), carbond disulfide ( $3 \mathrm{mmol}$ ) and 1,2-dibromoethane ( $1 \mathrm{mmol}$ ) in optimal reaction conditions (Table-2, entry 13$)$.

\section{Conclusion}

In summary, we have developed an one-pot and efficient method for the synthesis of 2-alkylthio-5-aryl-1,3,4-oxadiazole from acid hydrazides as substrate. Some of products are new and containing effective functional groups such as allyl, benzyl, acid and esters which they increase the volubility of products as intermediates in synthesis chemistry and biological activities. One-pot protocol, chemoselectivity, short reaction times, high yield, mild conditions, ease and safe handling are the main advantages of this method.

\section{ACKNOWLEDGEMENTS}

Financial support from Ilam University Research Council and the Iranian National Science Foundation (INSF, Grant No. 89003646) is gratefully acknowledged.

\section{REFERENCES}

1. J.P. Zhu and H. Bienayme, Multicomponent Reactions, Wiley-VCH: Weinheim (2005).

2. L.F. Tietze, A. Heins, M. Soleiman-Beigi and C. Raith, Heterocycles, 77, 1123 (2009)

3. S. Balalaie, M. Soleiman-Beigi and F. Rominger, J. Iran. Chem. Soc., 2, 319 (2005).

4. J. Wang, R. Wang, J. Yang, Z. Zheng, M.D. Carducci, T. Cayou, N. Peyghambarian and G.E. Jabbour, J. Am. Chem. Soc., 123, 6179 (2001).

5. C.J. Chen, B.-A. Song, S. Yang, G.-F. Xu, P.S. Bhadury, L.-H. Jin, D.-Y. Hu, Q.-Z. Li, F. Liu, W. Xue, P. Lu and Z. Chen, Bioorg. Med. Chem., 15, 3981 (2007)
6. A.G. Ghattas, H.A. El-sherief, A.E. Abdelrahman and A. M. Mahmoud, Pharmazie, 37, 410 (1982).

7. X.J. Zou, L.H. Lai, G.Y. Jin and Z.X. Zhang, J. Agric. Food Chem., 50, 3757 (2002).

8. S. Aboraia, H.M. Abdel-Rahman, N.M. Mahfouz and M.A. El-Gendy, Bioorg. Med. Chem., 14, 1236 (2006).

9. M.M. Burbuliene, V. Jakubkiene, G. Mekuskiene, E. Udrenaite, R. Smicius and P. Vainilavicius, Farmaco, 59, 767 (2004).

10. D.H. Boschelli, D.T. Connor, D.A. Bornemeier, R.D. Dyer, J.A. Kennedy, P.J. Kuipers, G.C. Okonkwo, D.J. Schrier and C.D. Wright, J. Med. Chem., 36, 1802 (1993).

11. G.C. Ramaprasad, B. Kallurya, B.S. Kumar and R.K. Hunnur, Eur. J. Med. Chem., 45, 4587 (2010)

12. F. Aydogan, Z. Turgut, N. Olcay and S.S. Erdem, Turk. J. Chem., 26, 159 (2002).

13. D.A. Charistos, G.V. Vagenes, L.C. Tzavellas, C.A. Tsoleridis and N.A. Rodios, J. Heterocycl. Chem., 31, 1593 (1994).

14. C.A. Tsoleridis, D.A. Charistos and G.V. Vagenes, J. Heterocycl. Chem., 34, 1715 (1997).

15. M.S.R. Murty, R.V. Rao, K.R. Ram, N.R. Reddy, J.S. Yadav and B. Sridhar, Synth. Commun., 40, 2914 (2010).

16. M.G. Mamolo, D. Zampieri, L. Vio, M. Fermeglia, M. Ferrone, S. Pricl and G. Scialino, Bioorg. Med. Chem., 13, 3797 (2005).

17. A. Rutavicius, S. Valyulene and Z. Kuodis, Chem. Heterocycl. Comp., 38, 852 (2002).

18. N. Jain, D.P. Pathak, P. Mishra and S. Jain, J. Iran. Chem. Soc., 6, 77 (2009).

19. T. Akhtar, S. Hameed, N.A. Al-Masoudi, R. Loddo and P.L. Colla, Acta Pharm., 58, 135 (2008).

20. S.V. Bhandari, K.G. Bothara, M.K. Raut, A.A. Patil, A.P. Sarkate and V.J. Mokale, Bioorg. Med. Chem., 16, 1822 (2008).

21. M. Koparir, A. Çetin and A. Cansiz, Molecules, 10, 475 (2005).

22. R. Smicius, V. Jakabkiene, M.M. Burbuluiene and P. Vainlavicius, Monatsh. Chem., 133, 173 (2002).

23. A.P. Andrushko, A.M. Demchenko, A.N. Krasovskii, E.B. Rusanov, A.N. Chernega and M.O. Lozinskii, Russ. J. Gen. Chem., 71, 1754 (2001).

24. R. Patil and J.S. Biradar, Indian J. Chem., 38B, 76 (1999).

25. Q.-Z. Zheng, X.-M. Zhang, Y. Xu, K. Cheng, Q.-C. Jiao and H.-L. Zhu, Bioorg. Med. Chem., 18, 7836 (2010).

26. H.P. Shah, B.R. Shah and N.K. Undavia, Indian J. Chem., 37B, 180 (1998).

27. M. Saitoh, J.E. Kunitomo, Y. Kimura, H. Hayase, N. Kobayashi, T. Uchiyama, T. Kawamoto, C. Tanaka, D. Mol, D.R. Dougan, G.S. Textor, G.P. Snell and F. Itoh, Bioorg. Med. Chem., 17, 2017 (2009).

28. E. Hoggsth, J. Chem. Soc., 4811 (1952).

29. M.A. Khali, S.M. El-Khawass and M.G. Kassem, Sci. Pharm., 48, 344 (1980).

30. M. Belkadi and A.A. Othman, ARKIVOC, 183 (2006).

31. D.E. Horning and J.M. Muchowski, Can. J. Chem., 50, 3079 (1972).

32. B. Movassagh and M. Soleiman-Beigi, Monatsh. Chem., 140, 409 (2009).

33. S.T. Kadam and S.S. Kim, Green Chem., 12, 94 (2010).

34. M. Zareef, R. Iqbal, B. Mirza, M.K. Khan, A. Manan, F. Asim and W.S. Khana, ARKIVOC, 141 (2008). 